\title{
Artificial neural network-based geometry compensation to improve the printing accuracy of selective laser melting fabricated sub-millimetre overhang trusses
}

Ruochen Hong ${ }^{1}$, Lei Zhang ${ }^{2}$, Joseph Lifton ${ }^{3}$, Stephen Daynes², Jun Wei², Stefanie Feih ${ }^{2, *}$ and Wen Feng $\mathrm{Lu}^{1, *}$

${ }^{1}$ Department of Mechanical Engineering, National University of Singapore, Singapore 117975

${ }^{2}$ Singapore Institute of Manufacturing Technology, 73 Nanyang Drive, Singapore 138634

${ }^{3}$ Advanced Remanufacturing and Technology Centre, 3 Cleantech Loop, Singapore 637143

\author{
Published in: \\ Additive Manufacturing, 101594, 2020.
}

* Corresponding authors: S. Feih (feihs@simtech.a-astar.edu.sg) and W.F. Lu (mpelwf@nus.edu.sg) 


\begin{abstract}
Selective laser melting processes deposit and join metal powders to near net shape in a layerby-layer manner. The process of melting and re-solidification of several layers of deposited material can result in geometric deviations, and the impact is particularly significant for submillimetre structures oriented at a wide range of overhang angles with respect to the building platform. This paper assesses and benchmarks the capabilities of a neural network-based geometric compensation approach for truss lattice structures with circular cross-sections. The neural network method is capable to generate free-form cross-sections with enhanced geometric freedom for compensation compared to more established analytical compensation approaches limited to predefined geometric shapes. For neural network training, lattice dome structures composed of trusses with different overhang angles were designed and printed by selective laser melting and measured via X-ray computed tomography, resulting in point cloud data sets containing more than 20,000 data points for each overhang angle. For experimental validation, neural network-compensated dome structures were benchmarked against dome structures with elliptical parameter compensation. Results show that the neural network compensated lattice trusses achieve higher printing dimensional accuracy compared to the uncompensated structures and those compensated based on elliptical parameter estimates.
\end{abstract}

Key words: Selective laser melting, Geometric compensation, Artificial neural network 


\section{Introduction}

Additive manufacturing (AM) is a manufacturing technology in which components are fabricated in a layer-by-layer additive process using a sliced Computer-Aided Design (CAD) model based on the desired geometry. The AM process of metal powder bed fusion (PBF) [1] can produce near net shape complex metallic parts with mechanical properties comparable to wrought counterparts [2]. With the advance of AM technologies for metallic materials, lightweight lattice structures have received significant research interests in recent years [3][4]. A lattice structure consists of internal architectures that can be engineered to achieve desired multifunctional performance such as high strength-to-weight ratios [5], negative Poisson's ratios [6], energy absorptions [7], heat transfer [8], and biomedical functions [9][10].

Despite the continuous capability improvements of PBF techniques for manufacturing complex metallic parts with advanced design freedom, deviations generally occur between the asdesigned and as-printed measured dimensions, especially for sub-millimetre features. The causes of printing imperfections and potentially printing failure are manifold as the process of melting and re-solidification of several layers of deposited material generally results in high residual stresses and related geometric distortions [11]. Geometric printing imperfections of AM fabricated lattices can be divided into two broad categories: random and systematic. Random imperfections of AM fabricated lattices exist in a form of statistical distributions such as thickness variations and layer offsets, and cannot be eradicated via geometric compensation [12],[13]. In contrast, systematic imperfections [14] of geometric deviations are repeatedly observed from every batch fabrication with a stable trend. Systematic imperfections of PBF printed parts are a result of coupled effects of file processing [15], process parameters [16] and powder characteristics [17]. Conventionally, where features with large overhang angles are required, support structures are printed to support the structure during solidification [18]. However, due to the fine and intricate features of lattices, it becomes impractical to remove support structures without inflicting structural damage. Metallic lattices fabricated by PBF without adding support structures have been shown to exhibit increased geometric deviations with increasing overhang angles [19][20]. The geometric imperfections, both random and systematic, are exceptionally pronounced for sub-millimetre lattices at large overhang angles.

To characterise the imperfect geometries of PBF printed lattices, X-ray computed tomography (XCT) is often used. XCT is a non-destructive scanning method to obtain micro-level spatial resolution 3D models and 2D truss cross-sections for material distribution and geometric analysis [21]. Parametric geometry fitting methods such as circular and elliptical fitting are widely used to quantify the dimensions of printed truss cross-sections. Systematic imperfections including truss over-sizing/under-sizing and elliptical cross-sectional distortion are found depending on printing overhang angles [22]. It has been reported that size deviation is most severe at overhang angles from $35^{\circ}$ to $45^{\circ}$, while circularity becomes poorer with increasing printing overhang angles [23][24].

Research efforts have focussed on minimising geometric imperfections of PBF printed lattices in mainly two areas: AM process optimisation and geometric compensation. For selective laser 
melting (SLM) processes, the influences of laser power, scanning speed, contour layers, hatch spacing and layer thickness of powder on the geometric quality of lattices were investigated and optimised [12][25][26]. Truss dimension accuracy and repeatability can be improved to a certain extent with optimised SLM process parameters. The influences of process parameters on part quality are determined for fine-tuning and optimisation via a generally costly experimental test matrix [27][28][29]. Still, process optimisation cannot fully eliminate geometric imperfections due to inherent process limitations. For example, the optimised parameters are generally applied for all trusses; the overhang angle, size and shape dependency therefore cannot be considered. In addition, with optimised process parameters the printed trusses still show deviations from the as-designed geometry for larger overhang angles, such as non-circular cross-sections, due to the penetration of the heat source into the layers below the current build layer [27].

Geometric compensation methods have been developed by researchers to further eliminate systematic imperfections. These methods measure the deviations between the as-designed and as-printed parts and generates compensated CAD models for printing while keeping the optimised process parameters fixed. The printed geometries from compensated models are expected to have minimum deviations to the as-designed lattices, i.e. circular strut crosssections of the correct area. The build orientation dependency of printed lattices is more easily addressed with this method as different compensation rules can be generated according to the respective overhang angles. In lattice compensations, a geometric parameterisation, e.g. circular and elliptical [30][31], is often used for as-design, as-printed, and compensated strut cross-sections, and then compensation of fabrication deviations is converted to an error minimisation problem. In terms of compensation methods, shrinkage compensation factors [32] are used in practice to apply compensation uniformly to the entire product or to apply different factors to the CAD model for each section of a product. However, uniform expansion or shrinkage may not fully address geometric deviations if the as-printed and as-designed parts result in highly dissimilar geometries. Circular or elliptical parameterisations of strut crosssections have been adopted for deviation prediction and compensation. Huang and co-workers [30-32,39] improved the offline shape shrinkage compensation using Fourier series parameterisation. The authors started from compensating cylindrical shapes to including more complex shapes such as polyhedrons [33], and further established a 3D geometric compensation and derivation of the optimal amount of compensation [34]. They also adopted polygon approximation with local compensation and circular approximation with selective corning to predict deviations of free-form shapes. However, with pre-defined cross-sectional shape parametrisation for pattern fitting and compensation, highly irregular shapes may not be captured accurately.

Artificial neural networks (ANNs) are a non-linear statistical data modelling tool which can be used to model highly non-linear relationships between input and output. Studies have shown that ANN has better performance in handling continuous variable features from 2 to 25 for prediction in regression problems than other supervised models such as Naïve Bayes, K-nearest neighbours and decision tresles. [35][36] ANNs are well known for their capacity to describe highly non-linear problems, thus showing a high potential applicability in ecological systems 
[37] and have recently been explored for additive manufacturing process improvements to minimise part distortions during printing [38] [39][40]. There are few research studies using ANN for geometric compensation [34,37]. Chowdhury et al. [41] used ANN based on a Levenberg-Marquardt variant of the error backpropagation algorithm to generate compensated designs for a 3D free-form shaped part using data generated by numerical simulation. Experimental validation is lacking yet having this additional information would be a stronger substantiation for the proposed mechanism. Noriega et al. [42] adopted the ANN algorithm for fused deposition modelling models and characterised the printing error of square prisms using coordinate measuring machine, achieving 30\% (internal dimensions) and 50\% (external dimensions) improvement on the dimensional accuracy. While the results look promising to vertical-oriented square prisms, the approach has not been applied to lattice structure where overhang angle constraint is the major challenge.

The research presented in this paper develops and applies an ANN-based compensation method to minimise the systematic imperfections of SLM printed lattices with sub-millimetre overhang trusses. The ANN model is developed based on a multilayer perceptron (MLP) structure with one hidden layer, capable to generate compensated free-form cross-sections in a polar coordinate system. Purpose designed dome-type lattice structures with circular and elliptic cross-sections and 5 discrete overhang angles were printed by SLM and then characterised via XCT. More than 20,000 data points were used for ANN model training. Results show that printing of the ANN-compensated shape leads to significantly improved accuracy for submillimetre lattices trusses at different overhang angles in terms of the size, area and aspect ratio of cross-sections.

\section{Methodology}

This section outlines the methodology for printing deviation characterisation and compensation approaches for the truss-based lattice structures. Lattice dome structures were designed, fabricated (see Section 2.1) and characterised via XCT as outlined in Section 2.2. Two compensation strategies are adopted: (1) A direct compensation scheme is used whereby the cross-section of each strut is assumed to be elliptical, this approach is adopted from [43] for benchmarking purposes. The dimensions of as-designed circles and as-printed ellipses are numerically related via closed-form analytical expressions as outlined in Section 2.3. The success of the direct compensation is directly related to the goodness of the elliptical fit assumption for the as-printed design. This will be further discussed in Section 4.2 and Section 4.3. (2) A free-form shape compensation methodology using ANN training and compensation is outlined in Section 2.4. Point cloud data sets of the as-designed models and as-printed structures are used for training of the ANN model. The trained ANN model then generates the compensated CAD design. Simulation-based validation studies of the ANN approach are performed with equation-generated data in Section 3. Section 4.3 then highlights results based on the experimental data. For the latter validation, compensated designs are then printed, characterised and compared with the target design for validation. The flowchart of this work is illustrated in Figure 1. 


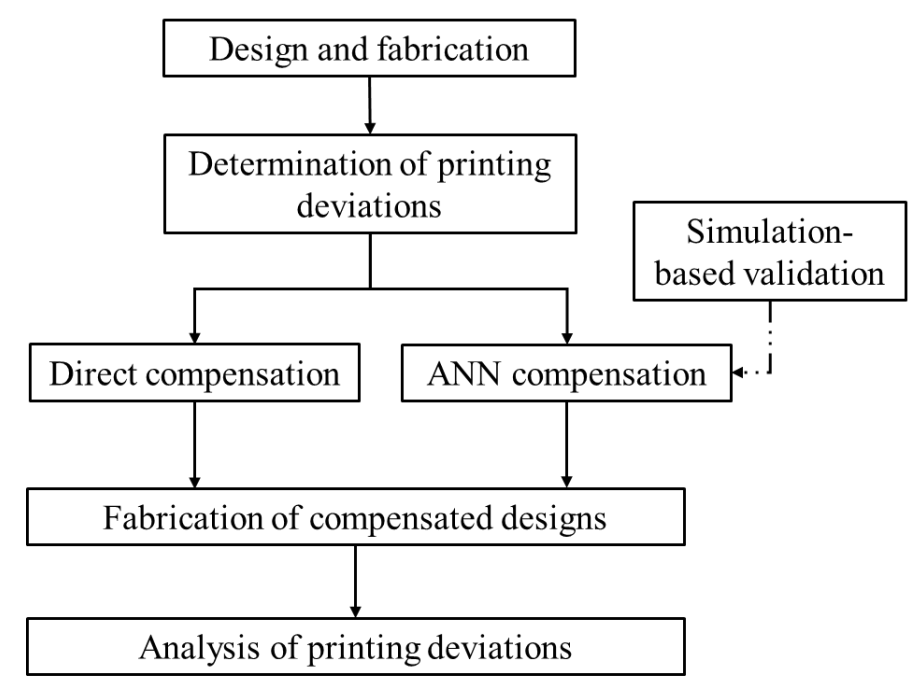

Figure 1: The flowchart of this work. Design and fabrication of specimen; characterisation of printing deviations; compensation using two methods; fabrication of compensated design; as well as comparison of results.

\subsection{Design and fabrication of lattice dome structures}

Dome structures composed of circular trusses were inspired by the spider-web geometry prospsed by Bagheri et al. [44]. The dome is a compact lattice structure designed for investigating the print accuracy of circular trusses orientated at five build overhang angles $\left(\theta_{1-5}=0^{\circ}, 15^{\circ}, 30^{\circ}, 45^{\circ}, 60^{\circ}\right)$ and six platform orientation angles $\left(\alpha_{1-6}=0^{\circ}, 60^{\circ}, 120^{\circ}, 180^{\circ}\right.$, $240^{\circ}, 300^{\circ}$ ) with respect to the respective platform orientations as shown in Figure 2. The length of each strut segment is $4.7 \mathrm{~mm}$. The printing response with respect to the overhang angle is influenced by the limited support of the powder bed as well as the spot size and remelting depth of the laser heat source [14]. In contrast, the printing response for the platform orientation is influenced by the recoater direction and gas flow [45]. Dome structures with circular and elliptical trusses were designed using Solidworks Version 2017 and later exported to stereolithography (STL) file format. The details of each dome design are shown in Table 1. Two specimens were printed for each design.

\begin{tabular}{llccccc}
\hline Overhang angle & & $0^{\circ}$ & $15^{\circ}$ & $30^{\circ}$ & $45^{\circ}$ & $60^{\circ}$ \\
\hline Design 1 & $r_{\text {design }}(\mathrm{mm})$ & 0.300 & 0.300 & 0.300 & 0.300 & 0.300 \\
Design 2 & $a_{\text {design }}(\mathrm{mm})$ & 0.295 & 0.310 & 0.320 & 0.320 & 0.330 \\
& $b_{\text {design }}(\mathrm{mm})$ & 0.295 & 0.290 & 0.280 & 0.280 & 0.275 \\
\multirow{2}{*}{ Design 3 } & $a_{\text {design }}(\mathrm{mm})$ & 0.298 & 0.315 & 0.300 & 0.295 & 0.300 \\
& $b_{\text {design }}(\mathrm{mm})$ & 0.297 & 0.295 & 0.255 & 0.245 & 0.240 \\
\hline
\end{tabular}

Table 1: As-designed dimensions of dome specimens. Design 1 is of circular truss with

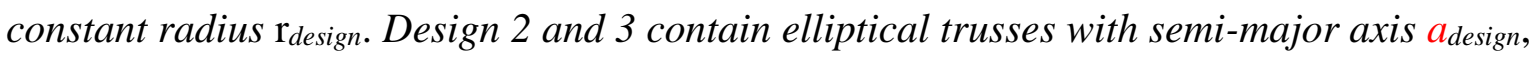
and semi-minor axis $b_{\text {design. }}$ 


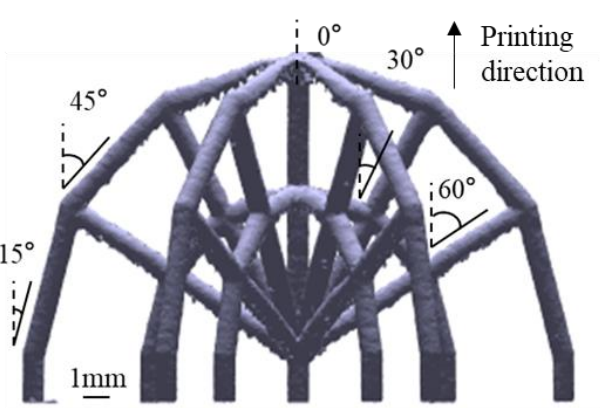

(a)

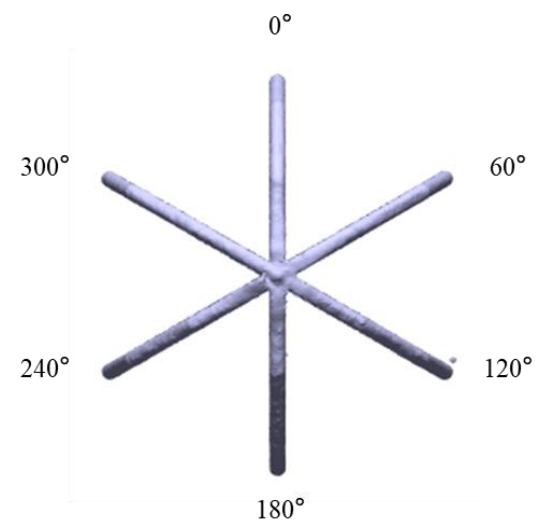

(b)

Figure 2: Automated profile generation of point cloud surface data obtained from X-ray CT scanned images. (a) Overhang angles $\left(\theta=0^{\circ}, 15^{\circ}, 30^{\circ}, 45^{\circ}, 60^{\circ}\right)$ are measured from the vertical (upright) printing direction. Each test specimen contains six trusses for each unique overhang angle (except for angle of $0^{\circ}$ ). (b) Visualisation of the six platform orientation angles $\left(\alpha=0^{\circ}, 60^{\circ}, 120^{\circ}, 180^{\circ}, 240^{\circ}, 300^{\circ}\right)$ with respect to powder bed.

The domes were printed from maraging steel powder [46] using SLM on an EOS M290 system. The SLM process parameters shown in Table 2 were optimised based on preliminary process optimisation for bulk material properties and kept constant for all printed lattice structures in this work. Additional optimisation of process parameters for truss structures is of course possible, but was not undertaken here as the research focus is placed on the validation of compensation schemes.

\begin{tabular}{cc}
\hline EOS M290 process parameter & Value \\
\hline Laser spot size $(\mu \mathrm{m})$ & 80 \\
Layer thickness $(\mu \mathrm{m})$ & 40 \\
Scanning speed $(\mathrm{mm} / \mathrm{s})$ & 960 \\
Laser power $(\mathrm{W})$ & 285 \\
Hatch distance $(\mu \mathrm{m})$ & 110 \\
\hline
\end{tabular}

Table 2: Process parameters of the SLM process

\subsection{Dimensional measurement}

Dimensional measurements of the samples were performed using XCT. The CT system used is developed in-house at the Singapore Institute of Manufacturing Technology (SIMTech). The system features a tungsten reflection target $225 \mathrm{kV}$ X-RAY WorX X-ray source and a $0.2 \mathrm{~mm}$ pixel pitch Perkin Elmer XRD 1621 flat panel detector with 2048 by 2048 pixels. The acquired $\mathrm{X}$-ray images were reconstructed into a 3D volumetric model using Volume Graphics VGStudio Max 3.1 (Volume Graphics GmbH, Germany). Surface determination was performed using the advanced method using the default settings and the surface model was converted to STL format and exported using the default settings in VGStudio. 
The strut dimensions were charaterized with an automated MATLAB in-house procedure as seen in Figure 3 (a). The procedure enabled an automated rotate/translate of the 3D STL model while isolating the strut of interest, followed by slicing of the point cloud data set. The point cloud is then used for ANN processing and non-linear least squares elliptical fitting along the isolated length of the strut for the parametric model and print deviation visualisation. Figure 3(b) shows the as-printed cross-section from the top view of the CT-generated STL model as well as representative printed shapes with respect to overhang angles, hereby indicating the increasing print deviation and resulting shape distortion as the overhang angle increases. The local distortion due to the melt pool capturing additional metallic powder particles on the underside of the truss during the reheating process of previous built layers for larger overhang angles results in significant distortions in the geometry as shown by the slice cuts along the truss.
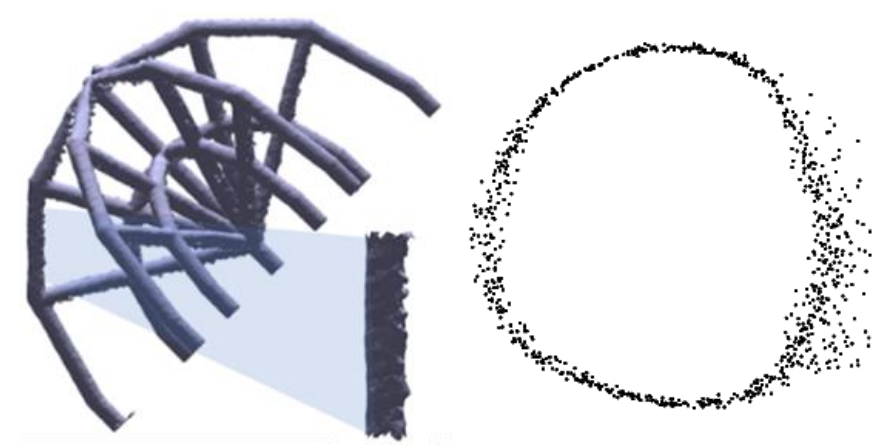

(a)

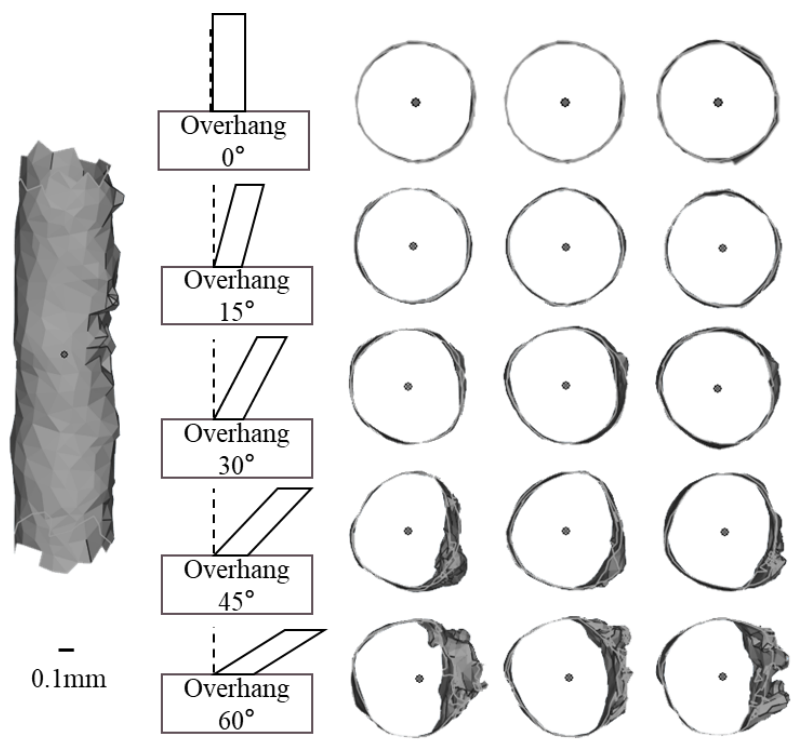

(b)

Figure 3: In-house procedure to automate print dimension characterisation: a) Rotation, transformation, isolation, slicing and generation of point cloud data sets for each truss and $b$ ) top view of as-printed truss sections showing increasing print deviations from round design geometry with larger overhang angles. 
For the evalution of dimensional accuracy and circularity of cross-sections, each strut was sliced into a series cross-sections and fitted with ellipses [47], along with major axis and minor axis values, and then further analysed and grouped depending on the overhang angle and platform orientation angle.

Elliptical fitting was undertaken as follows. Given a point cloud with $N$ samples $\left\{\left(x_{i}, y_{i}\right)\right\}, i=$ $1 \ldots N$, where $\left(x_{i}, y_{i}\right)$ are coordinates in cartesian coordinate system. The ellipse equation is described by a general conic with five parameters

$A x^{2}+B x y+C y^{2}+D x+E y+F=0$,

the distance from the point cloud to the ellipse is

$G=\sum_{i=1}^{n}\left(x_{i}^{2} A+x_{i} y_{i} B+y_{i}^{2} C+x_{i} D+y_{i} E+F\right)^{2}$

By minimising $G$, an fitted ellipse is obatained with semi-major and semi-minor axes $a_{\mathrm{fit}}$ and $b_{\text {fit }}$, respectively, where

$a_{\mathrm{fit}}=\sqrt{\frac{F}{A}}$
$b_{\mathrm{fit}}=\sqrt{\frac{F}{C}}$

\subsection{Parameter estimation method for compensation}

The parameter estimation (PE) method [43] is a direct method for compensation. The method adopts a shape parameterisation for as-printed and as-designed structures and establishes a closed-form correlation between them. The parameters for the correlation equations are generally obtained using numerical minimisation methods, such as the least squares method, for best fit to experimentally measured data. For strut lattices, the cross-sections of as-printed trusses are typically parameterised to fit circles [48] and ellipses [49] in Cartesian or polar coordinates.

In this work, the elliptical representation model is adopted due to enhanced geometric freedom compared to circular fitting. Printed cross-sections often appear elongated and preliminary investigations indicated a closer fit for an elliptical shape assumption compared to circular fitting, especially for larger overhang angles. The model relates as-designed circular trusses with a radius of $r_{0}$ and the as-printed cross-sectional shape by a parameterised equation for ellipses in polar coordinates:

$$
r(\varphi)=\frac{p_{x}\left(r_{0}+\Delta_{x}\right) p_{y}\left(r_{0}+\Delta_{y}\right)}{\sqrt{p_{x}^{2}\left(r_{0}+\Delta_{x}\right)^{2} \sin ^{2}(\varphi)+p_{y}^{2}\left(r_{0}+\Delta_{y}\right)^{2} \cos ^{2}(\varphi)}}
$$


where $r(\varphi)$ is the polar radius of as-printed cross-sections and $\varphi$ is the polar angle around the vertical direction for the transformed truss in its local coordinates. $p_{x}, p_{y}, \Delta_{x}$ and $\Delta_{y}$ are unknown parameters to be determined by fitting measured data in Eq. (1). To generate the compensated elliptical cross-section with semi-major axis $a_{\text {comp }}$ and semi-minor axis $b_{\text {comp }}$, the following formula is used:

$$
\begin{aligned}
& a_{\text {comp }}=\left(r_{0}+\Delta_{x}\right) \times p_{x} \\
& b_{\text {comp }}=\left(r_{0}+\Delta_{y}\right) \times p_{y}
\end{aligned}
$$

CAD files for dome structures with elliptical cross-sections were generated with SOLIDWORKS and converted to STL. Compensated dome structures were then printed for validation of the direct compensation approach.

\subsection{Neural network method for compensation}

While conventional topologies such as circular or elliptic shapes may be parameterised, the fitting process of highly irregular shapes such as observed for the as-printed cross-sections may be challenging with parameterisation methods. Numerical methods based on artificial neural network (ANN) training have the ability to correlate the as-designed and non-regular as-printed shapes without parameterisation, thereby enabling improved geometric fitting and compensation accuracy via a training process.

For benchmarking purposes, the XCT data used for the compensated design by parameter estimation method was also used for the compensated design by the proposed artificial neural network method. For the point cloud data origin determination, the centreline of the best-fit elliptical cylinder is approximated as the geometric centre of the point cloud. Point cloud datasets of different overhang angles are trained separately in the following. For any given specific overhang angle, the proposed ANN model has an input unit of a one by two vector, representing the polar angle and the polar radii from the geometric centre of the as-printed point cloud; the output unit is a one by one vector, representing the polar radii of the as-designed point cloud having the same polar angle as the input. The input data was pre-processed by converting the Cartesian coordinates of each point into polar coordinates and then normalised to a similar scale for radii and angle prior to training to ensure that each input parameter type has a comparable influence on the training model. As opposed to only the circular cross-section with design radius used in PE method, point cloud from all three designs in Table 1 are used for ANN training.

The artificial neural network set-up is based on multilayer perception (MLP) with one hidden layer as seen in Figure 4. According to Universal Approximation Theorem [50], a single hidden layer is sufficient for a multilayer perceptron to approximate any bounded continuous function. The ANN model architecture was simulated using the MATLAB Neural Network Toolbox, which is based on the Levenberg-Marquardt [51][52] variant of the error backpropagation algorithm for training the feed-forward network. An Error Backpropagation algorithm was used for minimising the error between the desired output and the calculated output of the 
network [53]. Assurance is needed as to validate the stability of ANN model and to prove that the model can interpret most of the patterns from the data correctly. A stable ANN model should be low in bias and variance. One significant advantage of utilizing XCT point cloud data sets is the large available data volume, represented by tens of thousands of data points, as shown in Table 3 . The trained model tends to be more robust for a dataset has a high number of observations.

\begin{tabular}{lccccc}
\hline Overhang angle & $0^{\circ}$ & $15^{\circ}$ & $30^{\circ}$ & $45^{\circ}$ & $60^{\circ}$ \\
\hline Point cloud size & 2432 & 23261 & 29922 & 34728 & 39566 \\
\hline
\end{tabular}

\section{Table 3: Size of point cloud used in neural network training}

No filtering is applied to the point cloud dataset determined from XCT. At the rougher downskin surface, more data points are generated from XCT to reconstruct the fine features in form of high roughness due to attached particles; therefore the down-skin surface has a larger point density than the up-skin surface. The ratio of point cloud size of down-skin surface to up-skin surface is largest for the overhang angle of $\theta=60^{\circ}$ with a ratio of 1.8. Since roughness is part of the true morphology of the as-printed specimens, utilizing the as-scanned point cloud data for ANN model training is preferable. To investigate the effect of the mismatched point density on the ANN training and compensation, the number of points in the down-skin surface are randomly sampled to match at a 1:1 ratio with that of in the up-skin surface, and the trained model results from the new datasets are compared with those of the as-scanned dataset. The results show that the imbalanced data point density in this study has negligible impact on the regression coefficients of the trained model and the resulting compensated cross-sections.

The original dataset was randomly split into a training, validation and test set, with $70 \%$ of data points grouped into the training set and the remaining $30 \%$ split equally between a validation and a test set. In each epoch (iteration), the weights are updated to accomodate data in the training set and susequently tested on the validation set. In the training process, the weights are initialised with some randomness, shifted by a small bias, and passed through the activation function. Weights are continuously evaluated and updated based on Error Backpropagation algorithm [54]. The training is terminated by adequate stopping criteria, in this case when a mean square error becomes less than 10-7, a maximum number of 1000 epochs is obtained or the mean squared error between the model-predicted cross section and the actual cross section of the validation set failed to decrease for six iterations. Once the trained model is obtained, we can input the desired cross-section, i.e. the circular cross-section with design radius, and the trained model will generate the shape of the compensated cross-section. The training process took a few minutes to complete on a computer with Intel(R) Core(TM) i5 CPU for all modelling and analysis.

A few rounds of parameter tuning in the model were tested with the experimental data to determine the optimal ANN architecture, the optimal number of neurons and the best training algorithm. An arcitecture of MLP 2-40-1 is selected, where the input layer has 2 neurons, the 
only hidden layer has 40 neurons and the output layer has one neuron. As seen in Figure 5, a neuron size of 40 in the hidden layer was selected to well balance the trade-off between computational time and accuracy.

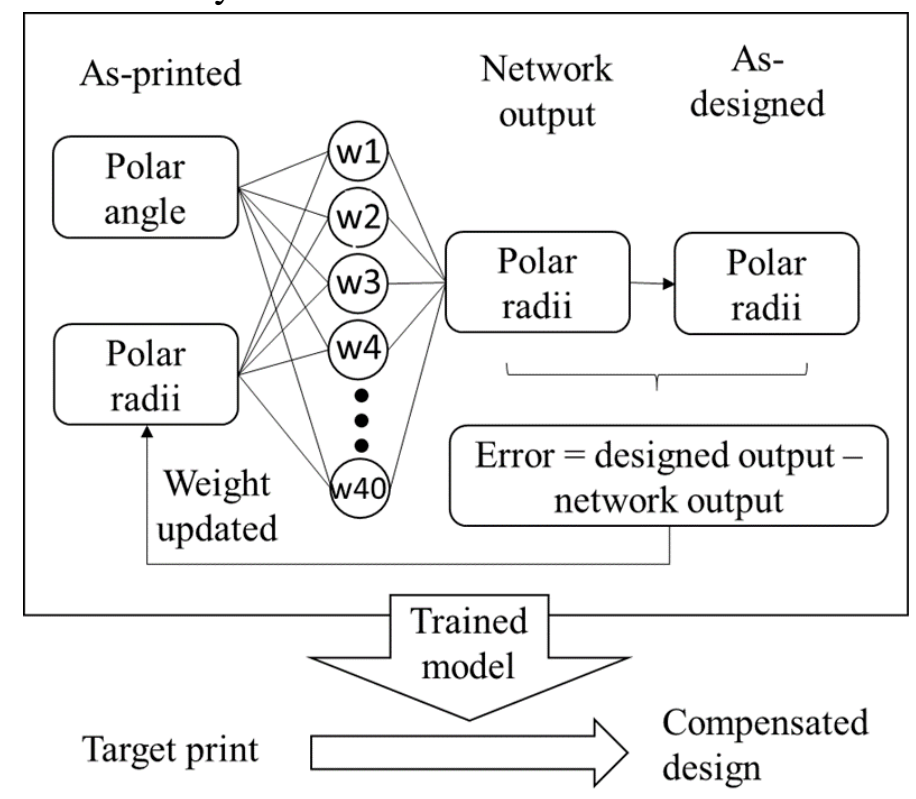

Figure 4: Artificial neural network flow chart for learning from printing deviation pattern and generating compensated design

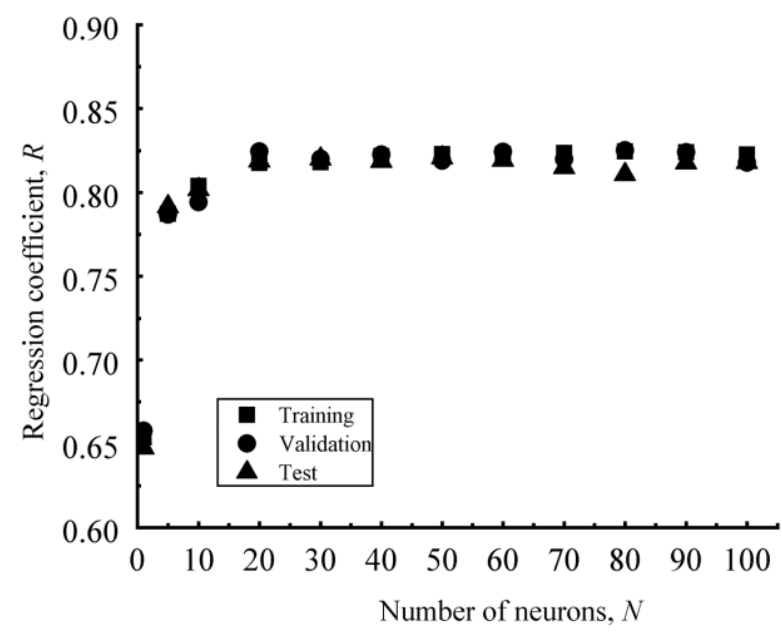

Figure 5: Regression coefficients of the training, validation and test set for the selection of optimum maximum number of neurons for ANN method for the selection of optimum number of neurons for ANN method. Data of overhang angle of $\theta=60^{\circ}$ for individual struts is used.

CAD files for dome structures with compensated free-form cross-sections were generated via SOLIDWORKS and converted to STL. Compensated dome structures were then printed for validation of the ANN compensation approach. 


\section{Simulation-based validation of ANN method}

\subsection{Input and training data generation}

For the purpose of validating the proposed ANN method to compensate artifically defined printing deviations, an equation-based relationship between the as-designed and as-printed cross-sections was assumed. A compensated design was then generated using the proposed ANN framework based on this pseudo-data generation.

If the as-designed cross-section radius is defined as $r(\varphi)$ in polar coordinates, the pseudo asprinted cross-sections $\tilde{r}(\varphi)$ is assumed to relate to $r(\varphi)$ by $f$ :

$$
\tilde{r}(\varphi)=f(r(\varphi))
$$

where $\varphi$ is again the polar angle. The trained ANN model is then able to predict the asdesigned shape $r(\varphi)$ based on the as-printed shape $\underline{r}(\varphi)$ as the input:

$$
\operatorname{ANN}(\tilde{r}(\varphi))=r(\varphi)
$$

Therefore, the function $\mathrm{ANN}$ is an inverse of $f$ such that $f$ generates the as-printed shape from a design, and ANN predicts the design from an as-printed shape as

$$
f(r(\varphi))=\mathrm{ANN}^{-1}(r(\varphi))
$$

Specifically, the compensated design $\bar{r}(\varphi)$ is obtained if the input of ANN is the expected asdesigned shape $r(\varphi)$. Input $\bar{r}(\varphi)$ in $f$, namely print the compensated design, results in a printed shape with the expected geometry $r(\varphi)$.

$$
\begin{aligned}
& \operatorname{ANN}(r(\varphi))=\bar{r}(\varphi) \\
& f(\bar{r}(\varphi))=f(\operatorname{ANN}(r(\varphi)))=f\left(f^{-1}(r(\varphi))\right)=r(\varphi)
\end{aligned}
$$

In this section, a perfect circle is used as the expected design with $r(\varphi)=r_{0}$. A Fourier series is adopted to produce regular variations for the pseudo as-printed cross-sectional shape represented by

$$
\tilde{r}(\varphi)=f(r(\varphi))=r(\varphi)+\sin \left(4 \varphi+\frac{3}{8} \pi\right)
$$

where $r_{0}$ is the as-designed radius. To consider the case of a single point on the boundary of a cross-section, $\left[\varphi, r_{0}\right]$ and $[\varphi, \tilde{r}(\varphi)]$ are the polar coordinates of the point on the as-designed and as-printed cross-sections, respectively. The input of the ANN model is then $[\varphi, \tilde{r}(\varphi)]$, and $\left[\varphi, r_{0}\right]$ is the desired constant output for the given input data values of $r_{0}=2,3,4$ and 5. 6184 data points in total were generated from Eq. (11), and 70\%, 15\%, 15\% of cloud points were used for the training, validation and testing data set, respectively, as per previous explanations in Section 2.4. 


\subsection{Results of the simulation-based validation studies}

The trained ANN model generates compensated geometry when provided with the input of the expected design with constant radius $r_{0}$. For a successfully formulated and trained ANN model, substituting the compensated cross-section into Eq. (9) should therefore result in the expected as-designed cross-section. Upon substituting the compensated shape into the pseudo as-printed cross-sections equation (Eq. (7)), the resulting $[\varphi, r]$ results in nearly perfect circles as shown in Figure 6. The slope of regression between as-designed and hypothetically as-printed point cloud is $R^{2}=1$ for all investigated radii, indicating that the proposed ANN model is capable of predicting the compensated cross-section of strut lattices to result in the desired as-printed shape. It is noted that a perfect compensation was possible in this case because the print deviation was captured via a perfectly fitted function. Section 4.2 further investigates the capabilities of the ANN compensation if scattered data is used for training purposes.

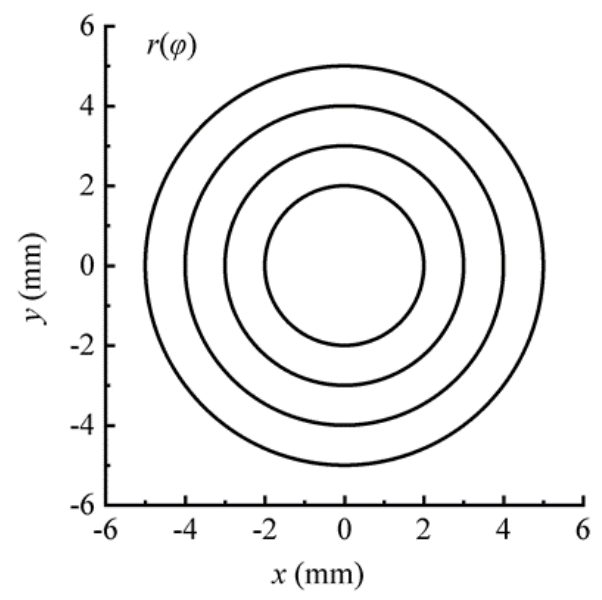

(a)

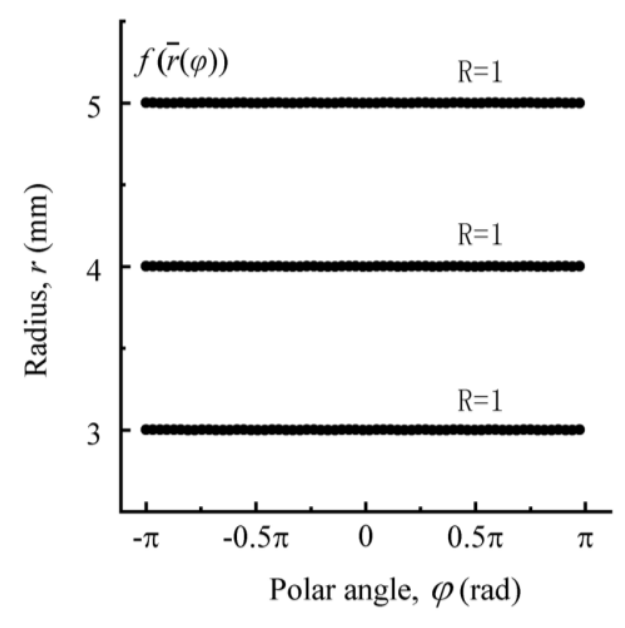

(c)

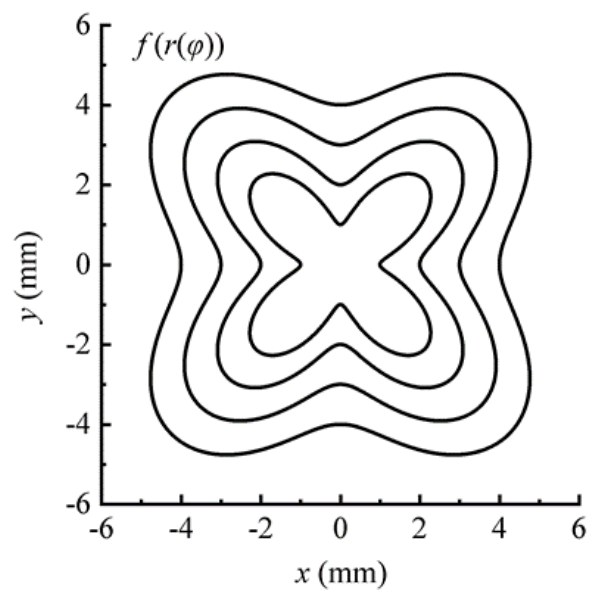

(b)

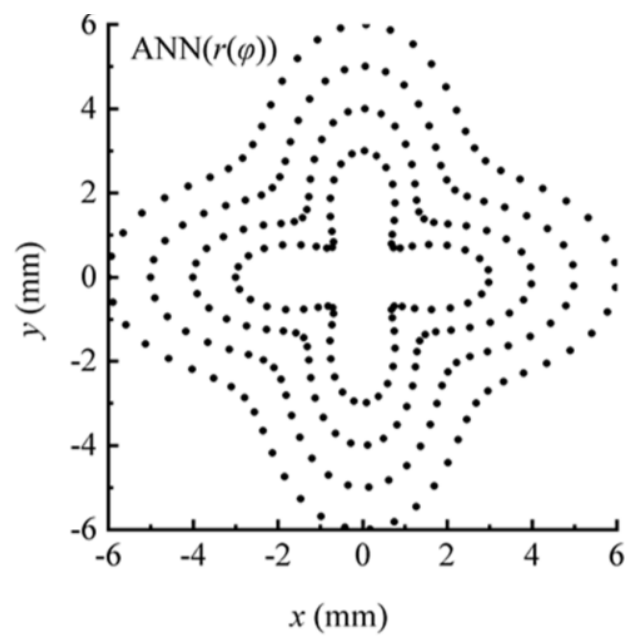

(d)

Figure 6: Simulation-based validation of neural network compensation method with (a) displaying the as-designed cross-section for different radii of $r_{0}=2,3,4$ and $5 \mathrm{~mm}$, (b) 
showing the pseudo-data generated by the Fourier series expression, (c) showing the compensated cross-section from point cloud data sets in $(a)$ and $(b)$ and $(d)$ goodness of prediction of c) and a).

\section{Results and discussions}

\subsection{Experimental charaterisation and fitting results (without compensation)}

Figure 7 shows the characterisation results following elliptical fitting for the as-printed crosssection for the different overhang angles and build platform orientations. The cluster of six columns per overhang angle refers to the results for the six truss build platform orientation angles with respect to the recoater direction. The presented results indicate that the influence of platform orientation is minimal, and input data for the compensation approaches were therefore averaged across the six strut platform orientations for the entire length of the same overhang angle. The dimensions were taken from the average over two dome specimens, which have high geometric conformity due to their cloes proximity on the building platform.

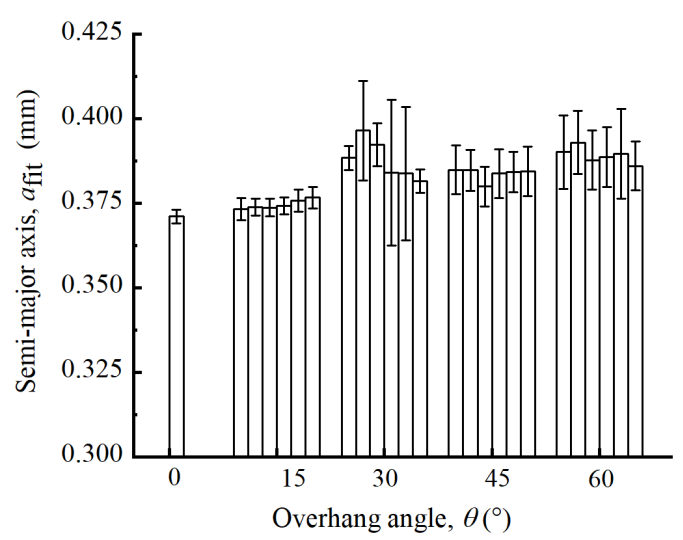

(a)

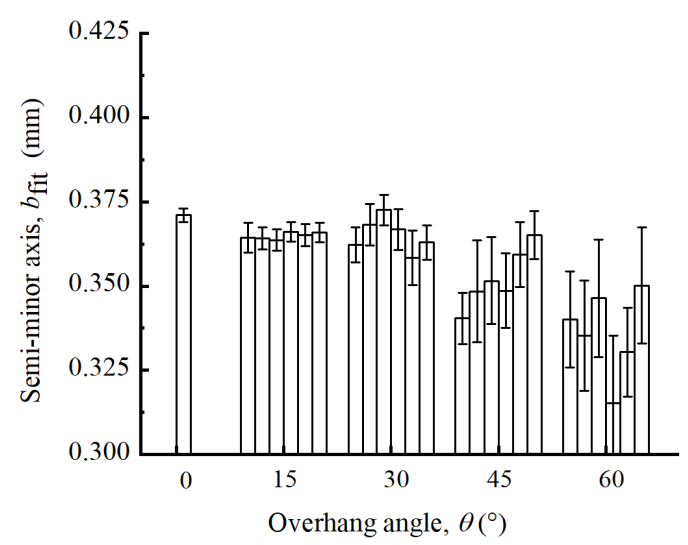

(b)

Figure 7: Geometry deviation patterns as a function of increasing overhang angles. a) Semimajor axis length of the fitted ellipse and $b$ ) semi-minor axis length of the fitted ellipse. The six different columns per build angle reflect results of build platform direction with respect to the recoater movement.

Consistent directional printing deviation patterns with respect to increasing overhang angle are also observed in Figure 7. It is seen that with an increase of overhang angle, print semi-major axis distance increases and print semi-minor axis distance descreases, resulting in an elongated shape with increased ellipticity. The as-printed cross-section is also consistently larger in area than the designed area. For the cross-sections of an overhang angle of $75^{\circ}$, thickness variation along the entire truss length is apparent and could not be averaged, thus this overhang angle was excluded from this study.

The presented patterns confirm the presence of systematic deviations in terms of semi-major and semi-minor as a function of the overhang angle. It is therefore postulated that these 
systematic data deviations can be minimised via compensation approaches by generating compensated designs and modified CAD models prior to printing.

\subsection{Evaluation of PE compensation results}

The parameters in Eq. (4) for PE compensation were calculated using the method of least squares. The input parameter set consisting of elliptical fitting axes $a_{\mathrm{fit}}$ and $b_{\mathrm{fit}}$ was considered as the mean of all six cross-sections with the same overhang angle. The parameters for the compensated designs were then obtained via calculation of semi-major and semi-minor axes as per Eq. (5). All resulting parameter values are shown in Table 4.

\begin{tabular}{ccccccccc}
\hline Overhang & $a_{\text {fit }}$ & $b_{\text {fit }}$ & $p_{x}$ & $p_{y}$ & $\Delta_{x}(\mathrm{~mm})$ & $\Delta_{y}(\mathrm{~mm})$ & $a_{\text {comp }}$ & $b_{\text {comp }}$ \\
\hline $0^{\circ}$ & 0.371 & 0.366 & 1.018 & 1.016 & 0.065 & 0.06 & 0.230 & 0.235 \\
$15^{\circ}$ & 0.372 & 0.365 & 1.018 & 1.016 & 0.065 & 0.059 & 0.229 & 0.236 \\
$30^{\circ}$ & 0.385 & 0.371 & 1.013 & 1.000 & 0.042 & 0.002 & 0.217 & 0.230 \\
$45^{\circ}$ & 0.381 & 0.360 & 1.016 & 1.002 & 0.051 & 0.006 & 0.220 & 0.240 \\
$60^{\circ}$ & 0.383 & 0.355 & 1.017 & 0.999 & 0.057 & -0.001 & 0.218 & 0.245 \\
\hline
\end{tabular}

Table 4: Offset and shrinkage parameters solved from Eq. (3) and Eq. (5) proposed in PE method. Parameters $a_{f i t}$ and $b_{\text {fit }}$ are averaged across the six different build platform orientations based on results presented in Figure 7.

Figure 8 shows the cross-sections of as-designed, as printed and PE-compensated design for the example of an overhang angle of $\theta=60^{\circ}$. As discussed previously, the as-printed crosssection is larger than designed and also elongated in the vertical direction. The compensated design area is therefore smaller than the designed cross-section, and the elliptical compensated shape is elongated in the horizontal direction.

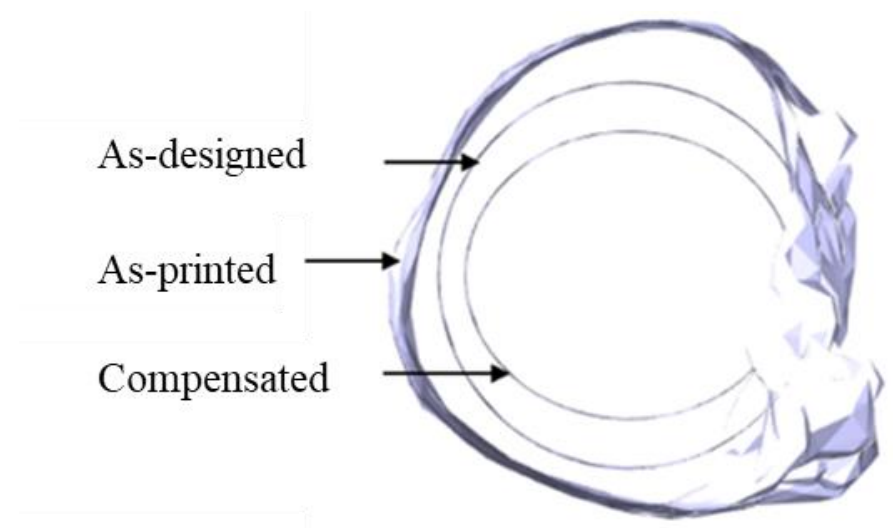

Figure 8: Cross-section through CAD design, actual print and compensated CAD with the parameter estimation method. Example here is given for an overhang angle of $\theta=60^{\circ}$.

Figure 9 shows the size of print across different overhang angles for the PE compensation method. Elliptical fitting is used to assess the roundness and area of the compensated as-printed trusses for quality evaluation. Semi-major axis and semi-minor axis lengths of the fitted ellipse 
and their respective standard deviations are compared. Slice-based and truss-based crosssections are examined, with the former being the average of 144 cross sections from six platform orientations and the latter being the average of all cross sections from the same truss. These two approaches are adopted to show the severity of cross-sectional variations across different layers due to roughness. Despite the similar results produced by the two approaches, the axis lengths of slice-based cross-sections are shown to exhibit increased standard deviations $(+37 \%)$ with increasing overhang angles compared to those of truss-based cross-sections. As of compensation, it can be seen that the direct elliptical compensation is successful at reducing the printed area closer to the design area. On average, the uncompensated print has a mean area percentage error $52 \%$ by slice as compared to the as-designed shape, and this error is reduced to around $1 \%$ of with PE. However, the direct compensation does not seem able to reduce the non-circular appearance of the cross-section for the non-zero overhang angles investigated in this work. The maximum aspect ratio of semi-major and semi-minor elliptical axes remains at 1.17 and continues to follow similar trends with increasing overhang angle.

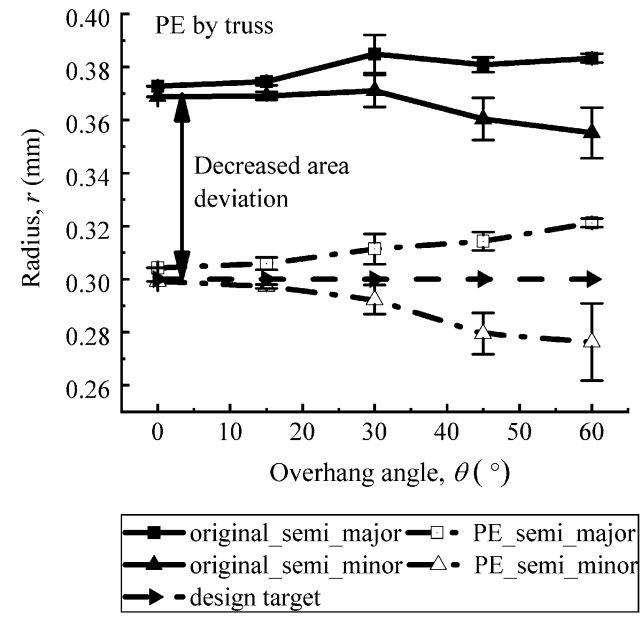

(a)

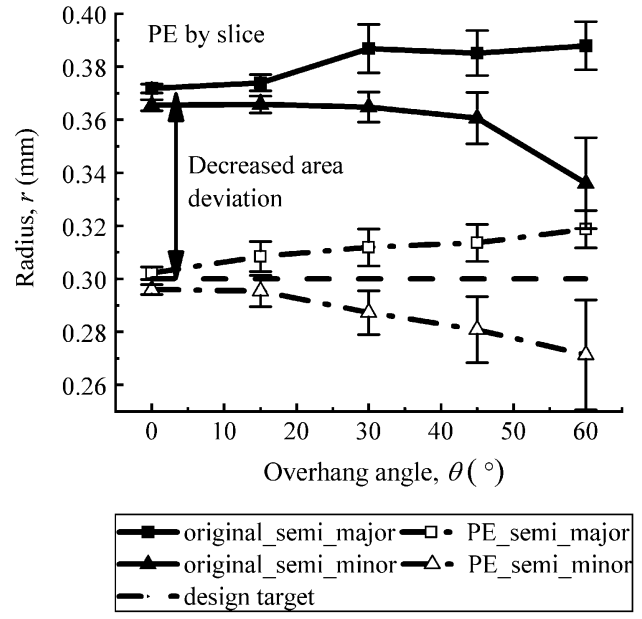

(b)

Figure 9: Comparison of compensated results for PE compensation. (a) shows the elliptical compensation accuracy as a function of overhang angle when evaluating the accuracy per truss with subsequent averaging for all trusses. (b) shows the elliptical compensation accuracy as a function of overhang angle when evaluating the accuracy per slice with subsequent averaging for all slices. The standard deviation was evaluated by averaging over 144 (by slices) and 12 (by trusses) cross-section profiles.

\subsection{Evaluation of ANN compensation results}

In contrast to the simulation-based points clouds where data points are located along distinct boundaries, the point cloud data sets of the as-printed structures contain scattered points that create partially overlapping regions. This is visualised in Figure 10. The curve section with positive $\mathrm{x}$ is the down-skin surface of the compensated cross section. The solid line represents fitted data output for every $2^{\circ}$ (equivalent to 180 data points), which were obtained from a trained model which is similar to the model in Section 2.4. Here a reversed dataset was used 
where input variables represent as-designed cross-sections and output variables represent the as-printed shapes. Figure 10 hence shows the capability of ANN in handling this scattered point cloud input well by deriving the respective fitted result of a free-form shape.

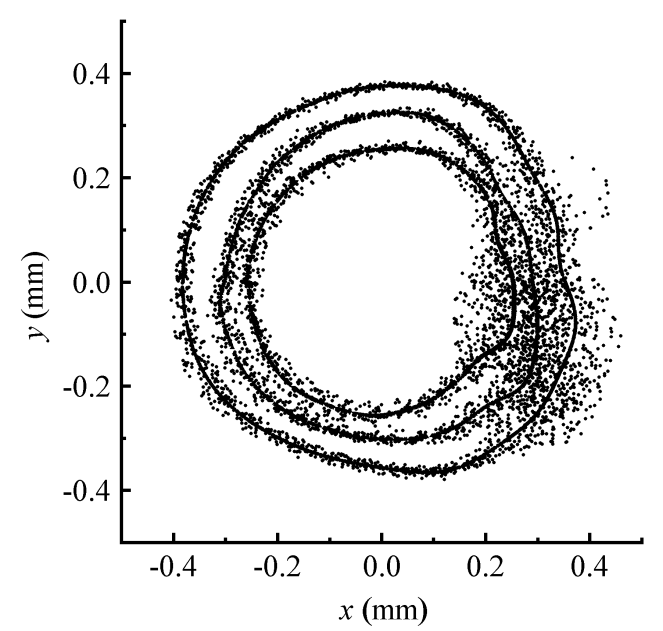

Figure 10: Cloud data set input for three radii and ANN output. For visualisation purposes, the data point output of the trained model is presented by solid lines based on discrete output information of 180 calculations (one for every $2^{\circ}$ ) to show how the ANN is able to fit free-from shapes out of scattered cloud point data sets. The curve section with positive $x$ is the down-skin surface of the compensated cross section.

Figure 11(a) shows the ability of the ANN model to generate compensated design input. The data input consists of the desired shape, a circle with radius of $0.3 \mathrm{~mm}$. The polar radii of the compensated shape are then generated by the ANN model, here visualised based on a solid line generated by 180 data points with $2^{\circ}$ angle spacing. The polar plot in Figure 11(b) highlights the highly irregular compensated cross-sectional shape obtainable with this method.

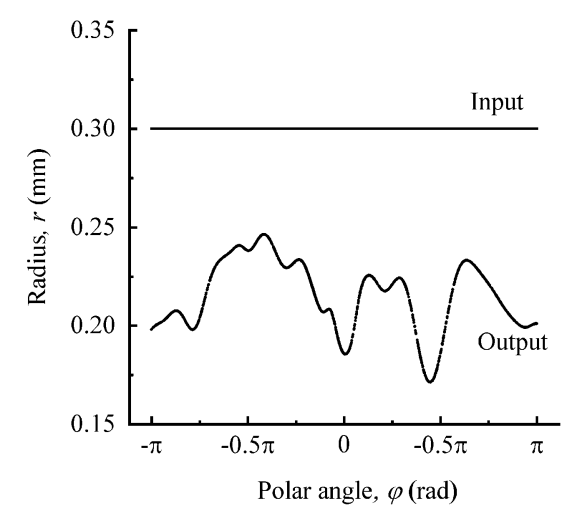

(a)

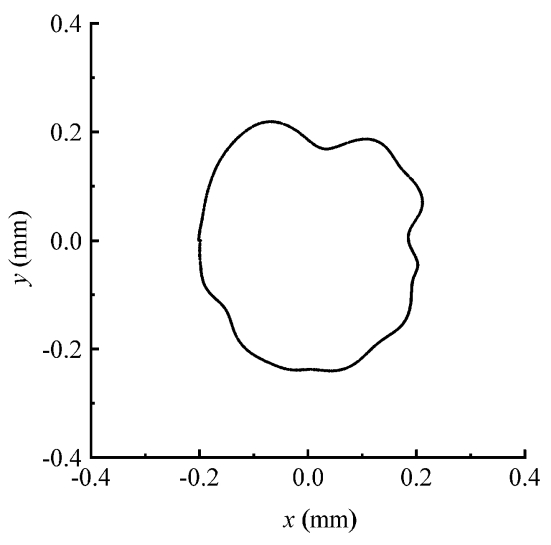

(b)

Figure 11: (a) Solid straight line at distance $=0.3 \mathrm{~mm}$ is the target polar radii and with the trained model a compensated design is generated and $(b)$ the cross section of the compensated 
shape. For visualisation purposes, the data point output of the trained model is presented by solid lines based on discrete output information of 180 calculations (one for every $2^{\circ}$ ).

Table 5 summarises the fitting accuracy results for ANN. The regression coefficient is the gradient of the best-fit line of the network output verus network target scatter plot. The regression coefficient decreases with inceasing overhang angle, yet all are close to 1 which implies a strong correlation between the experimental output and the model output. The accuracies of the training dataset and testing dataset indicate little difference. It shows that our network has a good generalisation performance. To further investigate the ANN stability, the method is deployed for 10 times, in an effort to examine how model performance varies depending on which observations end up in the training/validation set. The largest coefficient of variation (i.e. mean divided by standard deviation) for the area is $0.7 \%$.

\begin{tabular}{cccc}
\hline & Training & Validation & Test \\
\hline 0 & $1.000 \pm 0.000$ & $1.000 \pm 0.000$ & $1.000 \pm 0.000$ \\
15 & $1.000 \pm 0.000$ & $1.000 \pm 0.000$ & $1.000 \pm 0.000$ \\
30 & $0.977 \pm 0.000$ & $0.976 \pm 0.001$ & $0.975 \pm 0.001$ \\
45 & $0.903 \pm 0.001$ & $0.900 \pm 0.003$ & $0.901 \pm 0.001$ \\
60 & $0.822 \pm 0.001$ & $0.820 \pm 0.004$ & $0.819 \pm 0.004$ \\
\hline
\end{tabular}

Table 5: Regression coefficients of training, validation and test datasets for deviation modeling across different overhang angles. Regression coefficients are averaged over 10 runs.

Figure 12 shows the size of print deviation across different overhang angles for the ANN method. The printed cross-sections are again processed with elliptical fitting in the same manner to the PE compensation in Figure 9, resulting in semi-major and semi-minor axis length and their respective aspect ratio for visual quality evaluation. With ANN compensation, both semi-major axis length and semi-minor axis length are again drawn much closer to the target print reference line. The average area error is reduced to around 1\% with ANN compensation (similar to the PE method). However, the maximum aspect ratio between the semi-major and semi-minor axes also reduces significantly to 1.10 . 

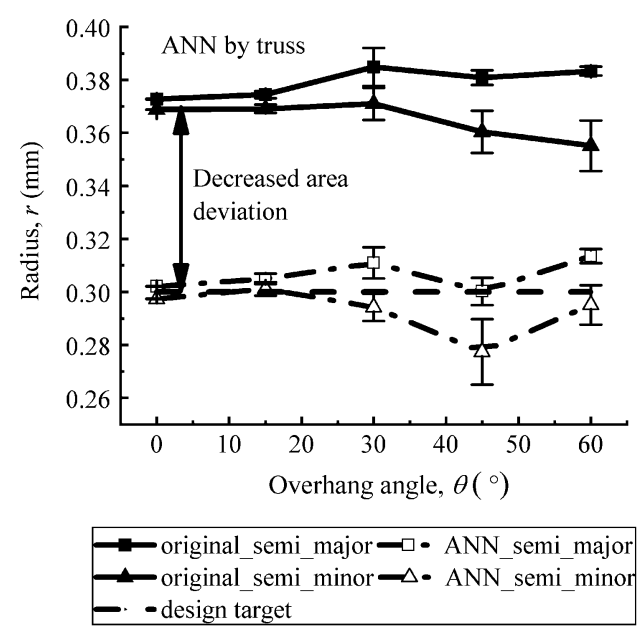

(a)

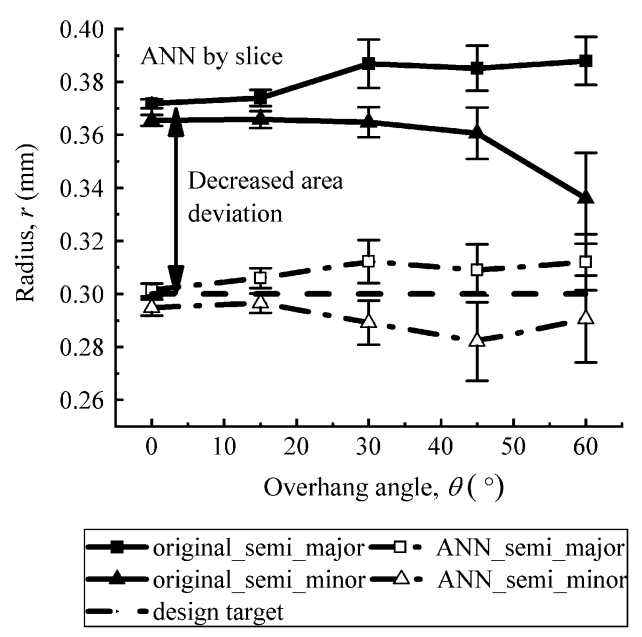

(b)

Figure 12: Comparison of compensated results for ANN compensation. (a) shows the elliptical compensation accuracy as a function of overhang angle when evaluating the accuracy per truss with subsequent averaging for all trusses. (b) shows the elliptical major and minor axis compensation accuracy as a function of overhang angle when evaluating the accuracy per slice with subsequent averaging for all slices. The standard deviation was evaluated by averaging over 144 (by slices) and 12 (by trusses) cross-section profiles.

\subsection{Comparison of $P E$ and ANN compensation results}

As discussed in the previous two sections, the ANN-compensated print results in a significant reduction of the elliptical elongation, especially for larger overhang angles. To further visualise this effect, Figure 13 shows the cross-sectional profiles of uncompensated, PE-and ANNcompensated prints. The ANN method results in a clearly superior free-form compensated geometry. The results demonstrate that the ANN compensation is better suited than the PE compensation in terms of achieving roundness of the printed lattice trusses, as demonstrated in this work up to an overhang angle of $\theta=60^{\circ}$. The increased performance and accuracy can be attributed to the ANN method's ability to generate free-form shapes to account for localised distortion, while PE compensation is restricted by its pre-defined elliptical parameterisation. 


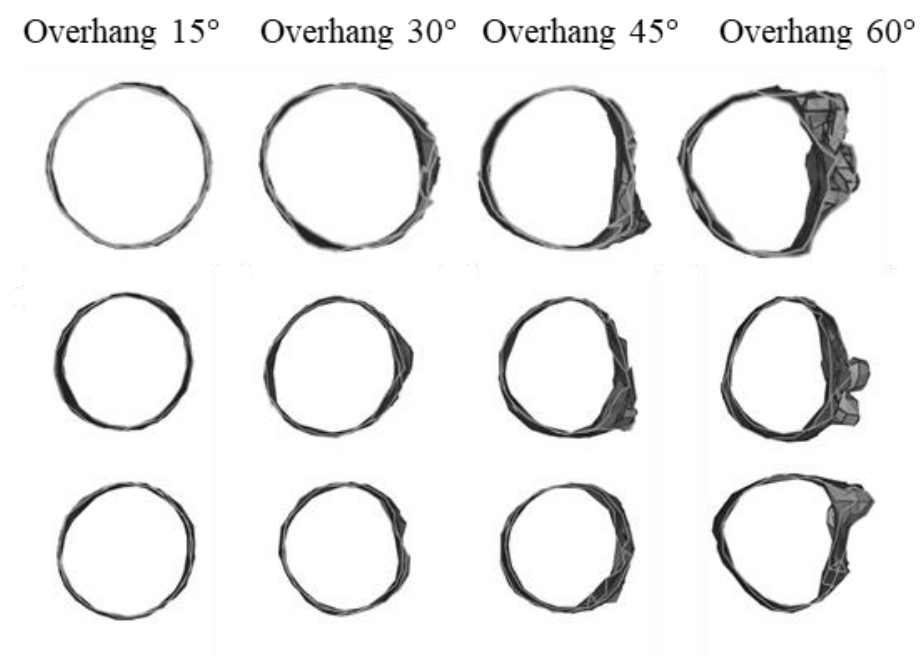

Figure 13: Cross-sections of uncompensated (top), PE- (middle) and ANN- (bottom) compensated print.

\section{Conclusions and future work}

In this work we propose an ANN based method to compensate SLM printed lattice structures and compare the results with an elliptical parameter estimation method. The proposed ANN model is based on an MLP structures with an input layer that connects to the two input variables, one hidden layer with 40 neurons and an output layer. The model is trained using 20000 to 40000 data points. Dome structures with varying strut diameters and overhang angles from $0^{\circ}$ to $60^{\circ}$ are SLM-printed and characterised by X-ray CT. The as-printed domes with noncompensated designs display oversizing and poor roundness for larger overhang angles.

Experimental results show that both PE and ANN approaches improve the cross-sectional area significantly, but the ANN method also results in a much improved roundness for the trusses, with a maximum axes aspect ratio of 1.10 as opposed to 1.17 for the PE method for the given wide range of overhang angles. The ANN compensation method is able to generate free-form lattice cross-sections to compensate local shape deviations more easily, thereby improving the agreement between the as-printed compensated structures and the as-designed ones.

The work presented via the ANN framework can be extended to allow for compensation of trusses at intermediate overhang angles not initially part of the dome design. It can also be extended to generate $3 \mathrm{D}$ compensation for overhang angles greater than $60^{\circ}$ where the assumption of uniform thickness along the length no longer holds valid.

\section{Acknowledgments}

This work is supported by the Agency for Science, Technology and Research (A*STAR) and the Science and Engineering Research Council (SERC) of Singapore through the Additive Manufacturing Centre (AMC) Initiative - SIMTech-led R\&D projects (SERC Grant no. 142 68 00088). 


\section{References}

[1] BSISO/ASTM 52910:2018 Additive manufacturing- Design- Requirements, guidelines and recommendations, Institution, Br. Stand. (2018).

[2] M. Koike, K. Martinez, L. Guo, G. Chahine, R. Kovacevic, T. Okabe, Evaluation of titanium alloy fabricated using electron beam melting system for dental applications, J. Mater. Process. Technol. 211 (2011) 1400-1408. https://doi.org/10.1016/j.jmatprotec.2011.03.013.

[3] D.S.J. Al-Saedi, S.H. Masood, M. Faizan-Ur-Rab, A. Alomarah, P. Ponnusamy, Mechanical properties and energy absorption capability of functionally graded F2BCC lattice fabricated by SLM, Mater. Des. 144 (2018) 32-44. https://doi.org/10.1016/j.matdes.2018.01.059.

[4] S. Daynes, S. Feih, W.F. Lu, J. Wei, Optimisation of functionally graded lattice structures using isostatic lines, Mater. Des. 127 (2017) 215-223. https://doi.org/10.1016/j.matdes.2017.04.082.

[5] L. Zhang, S. Feih, S. Daynes, Y. Wang, M.Y. Wang, J. Wei, W.F. Lu, Buckling optimization of Kagome lattice cores with free-form trusses, Mater. Des. 145 (2018) 144-155. https://doi.org/10.1016/j.matdes.2018.02.026.

[6] L. Yang, O. Harrysson, H. West, D. Cormier, Mechanical properties of 3D re-entrant honeycomb auxetic structures realized via additive manufacturing, Int. J. Solids Struct. 69-70 (2015) 475-490. https://doi.org/10.1016/j.ijsolstr.2015.05.005.

[7] N. Jin, F. Wang, Y. Wang, B. Zhang, H. Cheng, H. Zhang, Failure and energy absorption characteristics of four lattice structures under dynamic loading, Mater. Des. 169 (2019) 107655. https://doi.org/10.1016/j.matdes.2019.107655.

[8] M. Mazur, M. Leary, M. McMillan, J. Elambasseril, M. Brandt, SLM additive manufacture of $\mathrm{H} 13$ tool steel with conformal cooling and structural lattices, Rapid Prototyp. J. 22 (2016) 504-518. https://doi.org/10.1108/RPJ-06-2014-0075.

[9] N. Taniguchi, S. Fujibayashi, M. Takemoto, K. Sasaki, B. Otsuki, T. Nakamura, T. Matsushita, T. Kokubo, S. Matsuda, Effect of pore size on bone ingrowth into porous titanium implants fabricated by additive manufacturing: An in vivo experiment, Mater. Sci. Eng. C. 59 (2016) 690-701. https://doi.org/10.1016/j.msec.2015.10.069.

[10] G. Savio, R. Meneghello, G. Concheri, Optimization of lattice structures for additive manufacturing technologies, Adv. Mech. Des. Eng. Manuf. (2017) 213-222. https://doi.org/10.1007/978-3-319-45781-9_22.

[11] P.M.J. Kruth, P. Mercelis, J. Kruth, Residual stresses in selective laser sintering and selective laser melting, Rapid Prototyp. J. Vol. 12 (2006) 254-265. https://doi.org/10.1108/13552540610707013.

[12] F.A. List, R.R. Dehoff, L.E. Lowe, W.J. Sames, Properties of Inconel 625 mesh structures grown by electron beam additive manufacturing, Mater. Sci. Eng. A. 615 (2014) 191-197. https://doi.org/10.1016/j.msea.2014.07.051.

[13] Z. Chen, X. Wu, D. Tomus, C.H.J. Davies, Surface roughness of Selective Laser Melted Ti-6Al-4V alloy components, Addit. Manuf. 21 (2018) 91-103. 
https://doi.org/10.1016/j.addma.2018.02.009.

[14] H. Gong, K. Rafi, H. Gu, T. Starr, B. Stucker, Analysis of defect generation in Ti-6Al$4 \mathrm{~V}$ parts made using powder bed fusion additive manufacturing processes, Addit. Manuf. 1 (2014) 87-98. https://doi.org/10.1016/j.addma.2014.08.002.

[15] M. Taufik, P.K. Jain, Estimation and simulation of Shape deviation for Additive Manufacturing prototypes, Proc. ASME Des. Eng. Tech. Conf. Comput. Inf. Eng. Conf. 4 (2016). https://doi.org/10.1115/DETC2016-60035.pdf.

[16] B. Vayre, F. Vignat, F. Villeneuve, Identification on some design key parameters for additive manufacturing : application on Electron Beam Melting, Procedia CIRP. 7 (2013) 264-269. https://doi.org/10.1016/j.procir.2013.05.045.

[17] I. Baturynska, O. Semeniuta, K. Martinsen, Optimization of process parameters for powder bed fusion additive manufacturing by combination of machine learning and finite element method: A conceptual framework, Procedia CIRP. 67 (2018) 227-232. https://doi.org/10.1016/j.procir.2017.12.204.

[18] J.P. Järvinen, V. Matilainen, X. Li, H. Piili, A. Salminen, I. Mäkelä, O. Nyrhilä, Characterization of effect of support structures in laser additive manufacturing of stainless steel, Phys. Procedia. 56 (2014) 72-81.

https://doi.org/10.1016/j.phpro.2014.08.099.

[19] R. Wauthle, B. Vrancken, B. Beynaerts, K. Jorissen, J. Schrooten, J.P. Kruth, J. Van Humbeeck, Effects of build orientation and heat treatment on the microstructure and mechanical properties of selective laser melted Ti6Al4V lattice structures, Addit. Manuf. 5 (2015) 77-84. https://doi.org/10.1016/j.addma.2014.12.008.

[20] D. Wang, Y. Yang, R. Liu, D. Xiao, J. Sun, Study on the designing rules and processability of porous structure based on selective laser melting ( SLM ), J. Mater. Process. Tech. 213 (2013) 1734-1742. https://doi.org/10.1016/j.jmatprotec.2013.05.001.

[21] A. du Plessis, I. Yadroitsev, I. Yadroitsava, S.G. Le Roux, X-Ray Microcomputed Tomography in Additive Manufacturing: A Review of the Current Technology and Applications, 3D Print. Addit. Manuf. (2018) 227-247. https://doi.org/10.1089/3dp.2018.0060.

[22] U. Paggi, M. Sinico, L. Thijs, W. Dewulf, B. Van Hooreweder, Improving the dimensional accuracy of downfacing surfaces of additively manufactured parts, Eur. Soc. Precis. Eng. Nanotechnol. (2019).

[23] Z. Dong, Y. Liu, W. Li, J. Liang, Orientation dependency for microstructure, geometric accuracy and mechanical properties of selective laser melting AlSi10Mg lattices, J. Alloys Compd. 791 (2019) 490-500. https://doi.org/10.1016/j.jallcom.2019.03.344.

[24] X.Z. Zhang, H.P. Tang, M. Leary, T. Song, L. Jia, M. Qian, Toward Manufacturing Quality Ti-6Al-4V Lattice Struts by Selective Electron Beam Melting (SEBM) for Lattice Design, JOM. 70 (2018) 1870-1876. https://doi.org/10.1007/s11837-018-3030$\mathrm{X}$.

[25] C. Qiu, S. Yue, N.J.E. Adkins, M. Ward, H. Hassanin, P.D. Lee, P.J. Withers, M.M. Attallah, Influence of processing conditions on strut structure and compressive 
properties of cellular lattice structures fabricated by selective laser melting, Mater. Sci. Eng. A. 638 (2015) 228-231. https://doi.org/10.1016/j.msea.2015.04.074.

[26] S. Zhang, S. Dilip, L. Yang, H. Miyanaji, B. Stucker, P. City, Property Evaluation of Metal Cellular Strut Structures via Powder Bed Fusion AM, Solid Free. Fabr. Symp. (2015) 1410-1421.

[27] N. Béraud, F. Vignat, F. Villeneuve, R. Dendievel, Improving dimensional accuracy in EBM using beam characterization and trajectory optimization, Addit. Manuf. 14 (2017) 1-6. https://doi.org/10.1016/j.addma.2016.12.002.

[28] A.K. Sood, R.K. Ohdar, S.S. Mahapatra, Experimental investigation and empirical modelling of FDM process for compressive strength improvement, J. Adv. Res. 3 (2012) 81-90. https://doi.org/10.1016/j.jare.2011.05.001.

[29] G. Strano, L. Hao, R.M. Everson, K.E. Evans, Surface roughness analysis , modelling and prediction in selective laser melting, J. Mater. Process. Tech. 213 (2013) 589-597. https://doi.org/10.1016/j.jmatprotec.2012.11.011.

[30] K. Xu, T.H. Kwok, Z. Zhao, Y. Chen, A reverse compensation framework for shape deformation control in additive manufacturing, J. Comput. Inf. Sci. Eng. 17 (2017). https://doi.org/10.1115/1.4034874.

[31] S. Christoph, B. Fabian, J. Stephan, S. Christian, F.Z. Michael, Pre-compensation of Warpage for Additive Manufacturing, J. Mech. Eng. Autom. 6 (2016) 392-399. https://doi.org/10.17265/2159-5275/2016.08.002.

[32] Q. Huang, J. Zhang, A. Sabbaghi, T. Dasgupta, Optimal offline compensation of shape shrinkage for three-dimensional printing processes, IIE Trans. (Institute Ind. Eng. 47 (2015) 431-441. https://doi.org/10.1080/0740817X.2014.955599.

[33] H. Luan, Q. Huang, Predictive modeling of in-plane geometric deviation for 3D printed freeform products, IEEE Int. Conf. Autom. Sci. Eng. (2015) 912-917. https://doi.org/10.1109/CoASE.2015.7294215.

[34] Y. Jin, S.J. Qin, Q. Huang, Out-of-Plane Geometric Error Prediction for Additive Manufacturing, 2015 IEEE Int. Conf. Autom. Sci. Eng. (2015) 918-923.

[35] L.W.K. Setiono R., Pruned Neural Networks for Regression, in: Mizoguchi R., Slaney J. PRICAI 2000 Top. Artif. Intell. PRICAI 2000. Lect. Notes Comput. Sci., Springer, Berlin, Heidelberg, 2000.

[36] I.K. and M.T.G. M. Kayri, The performance comparison of Multiple Linear Regression, Random Forest and Artificial Neural Network by using photovoltaic and atmospheric data, 14th Int. Conf. Eng. Mod. Electr. Syst. (EMES), Oradea. (2017) 1-4. https://doi.org/doi: 10.1109/EMES.2017.7980368.

[37] S. Lek, J.F. Guégan, Artificial neural networks as a tool in ecological modelling, an introduction, Ecol. Modell. 120 (1999) 65-73. https://doi.org/10.1016/S03043800(99)00092-7.

[38] L. Di Angelo, P. Di Stefano, A neural network-based build time estimator for layer manufactured objects, Int J Adv Manuf Technol. (2011) 215-224. https://doi.org/10.1007/s00170-011-3284-8.

[39] J. Francis, L. Bian, Deep Learning for Distortion Prediction in Laser-Based Additive 
Manufacturing using Big Data, Manuf. Lett. (2019).

https://doi.org/10.1016/j.mfglet.2019.02.001.

[40] M. Zhao, G. Xiong, X. Shang, C. Liu, Z. Shen, H. Wu, Nonlinear deformation prediction and compensation for 3d printing based on CAE neural networks, IEEE Int. Conf. Autom. Sci. Eng. 2019-Augus (2019) 667-672. https://doi.org/10.1109/COASE.2019.8843210.

[41] S. Chowdhury, K. Mhapsekar, S. Anand, Part build orientation optimization and neural network based geometry compensation for additive manufacturing process, J. Manuf. Sci. Eng. Receiv. (2017). https://doi.org/10.1115/1.4038293.

[42] A. Noriega, D. Blanco, B.J. Alvarez, A. Garcia, Dimensional accuracy improvement of FDM square cross-section parts using artificial neural networks and an optimization algorithm, Int. J. Adv. Manuf. Technol. 69 (2013) 2301-2313. https://doi.org/10.1007/s00170-013-5196-2.

[43] L. Xu, Q. Huang, A. Sabbaghi, T. Dasgupta, Shape deviation modeling for dimensional quality control in additive manufacturing, Proc. ASME 2013 Int. Mech. Eng. Congr. Expo. (2013). https://doi.org/10.1115/IMECE2013-66329.

[44] Z.S. Bagheri, D. Melancon, L. Liu, R.B. Johnston, D. Pasini, Compensation strategy to reduce geometry and mechanics mismatches in porous biomaterials built with Selective Laser Melting, J. Mech. Behav. Biomed. Mater. 70 (2017) 17-27. https://doi.org/10.1016/j.jmbbm.2016.04.041.

[45] J. Kranz, D. Herzog, C. Emmelmann, Design guidelines for laser additive manufacturing of lightweight structures in TiAl6V4, J. Laser Appl. 27 (2015) S14001. https://doi.org/10.2351/1.4885235.

[46] Systems EOS GmbH - Electro Optical, Material data sheet EOS MaragingSteel MS1, (2011) 1-6.

[47] P.L. Rosin, A note on the least squares fitting of ellipses, Pattern Recognit. Lett. 14 (1993) 799-808. https://doi.org/10.1016/0167-8655(93)90062-I.

[48] S.Y. Yuen, C.H. Ma, An investigation of the nature of parameterization for the Hough Transform, J. Text. Inst. Trans. 30 (1997) 1009-1040. https://doi.org/10.1080/19447022408661298.

[49] A.S. Aguado, M.E. Montiel, M.S. Nixon, On using directional information for parameter space decomposition in ellipse detection, Pattern Recognit. 29 (1996) 369381. https://doi.org/10.1016/0031-3203(94)00096-4.

[50] K. Hornik, Approximation Capabilities of Multilayer Neural Network, Neural Networks. 4 (1991) 251-257.

[51] K. Neumann, A method for the solution of certain nonlinear control problems, Computing. 6 (1970) 249-263. https://doi.org/10.1007/BF02238810.

[52] D.W. Marquardt, An Algorithm for Least-Squares Estimation of Nonlinear Parameters, J. Soc. Ind. Appl. Math. 11 (1963) 431-441. https://doi.org/10.1137/0111030.

[53] D.E. Chauvin, Rumelhart, Backpropagation: Theory, Architectures, and Applications, Psychology Press, 1995. 
[54] D.E. Rumelhart, G.E. Hinton, R.J. Williams, Parallel distributed processing: Explorations in the microstructure of cognition, MIT Press, 1986. 